\title{
Combined All-Extremity High-Intensity Interval Training Regulates Immunometabolic Responses through Toll-Like Receptor 4 Adaptors and A20 Downregulation in Obese Young Females
}

\author{
Nakisa Soltani ${ }^{a}$ Sayed Mohammad Marandi ${ }^{a} \quad$ Mohammad Kazemi ${ }^{\mathrm{b}}$ \\ Nafiseh Esmaeilc \\ ${ }^{a}$ Department of Exercise Physiology, Faculty of Sport Sciences, University of Isfahan, \\ Isfahan, Iran; 'bepartment of Genetics and Molecular Biology, University of Medical \\ Sciences, Isfahan, Iran; ' Department of Immunology, School of Medicine, University of \\ Medical Sciences, Isfahan, Iran
}

\section{Keywords}

Exercise therapy · Immunoregulatory effects · Toll-like receptor $4 \cdot$ Obesity ·

Metainflammation

\begin{abstract}
Metainflammation and malfunctions of toll-like receptor 4 (TLR4) are related to obesity-induced immunometabolic morbidities. There are almost no studies relating exercise training to the TLR4 pathway and its adaptors and negative regulators. Thirty young women with obesity (exercise group and control group) were included in a 10-week all-extremity combined high-intensity interval training program. The immunomodulatory impacts of exercise on TLR4, its related adaptors (TIR domain-containing adaptor-inducing IFN- $\beta$ [TRIF], myeloid differentiation factor 88 [MyD88], and tumor receptor-associated factor 6 [TRAF6]), transcriptional factors (nuclear factor [NF]- $k B$ and interferon regulatory factor 3 [IRF3]), and negative regulator (A20) mRNA levels were assessed by real-time PCR. Also, the serum concentration of TLR4 final products (tumor necrosis factor $\alpha$ [TNF $\alpha]$ and interferon $\gamma$ [IFN $\gamma]$ ) was measured by ELISA. Cardiorespiratory and body composition parameters were tested, as well. There was a significant improvement in body composition and cardiorespiratory fitness. This intervention downregulated TLR4 (from $2.25 \pm 1.07$ to $0.84 \pm 1.01$ ), MyD88 (from $4.53 \pm 5.15$ to $1.27 \pm 0.88$ ), NF-KB (from $1.61 \pm 2.03$ to $0.23 \pm 0.39$ ), IRF3 (from $1.22 \pm 0.77$ to $0.25 \pm 0.36$ ), and A20 (from $0.88 \pm 0.59$ to $0.22 \pm 0.33$ ) levels and reduced the TNF $\alpha$ concentrations (from $22.39 \pm 11.43$ to
\end{abstract}

Nafiseh Esmaeil

Department of Immunology, School of Medicine

Isfahan University of Medical Sciences, Hezar Jerib St.

Isfahan 81746-73461 (Iran)

nafesm5 @ gmail.com
Sayed Mohammad Marandi

Department of Exercise Physiology

Faculty of Sport Sciences, University of Isfahan

Azadi Sq., Isfahan 81746-73441 (Iran)

smmarandi2001@yahoo.com 
Soltani et al.: Exercise Training, TLR4 Pathway, and Negative Regulators

$6.26 \pm 5.31$ ) significantly in the exercise group, while no statistically significant change was found in TRIF and TRAF6 expression and IFN $\gamma$ circulating levels. It is concluded that long-term exercise modifies the inflammatory pathways and modulates the immune function at the early stages of inflammation initiation in circulating immune cells. Accordingly, we suggest time-efficient exercise protocols as a possible therapy approach for the prevention of M1 polarization.

(C) 2020 The Author(s)

Published by S. Karger AG, Basel

\section{Introduction}

Modern lifestyle-induced immunometabolic disorders, like insulin resistance (IR), type 2 diabetes, cancer, autoimmune diseases, and neurodegeneration, have become a global burden because they promote the premature death $[1,2]$. Due to the strong association between obesity, metabolic disturbances, and mortality risk, obesity has become a causal element in the etiology of these chronic diseases [2-4]. Although obesity is a multifactorial state, chronic low-grade systemic inflammation, known as metainflammation, is a hallmark that partly links all of these disturbances through promotion of the activation and interactions of many inflammatory pathways in multiple cells [1]. During the development of obesity, pathologic expansion of adipocytes in adipose tissue (AT) induces hypoxia and adipocytes necrosis, which act as initiators of chronic low-grade systemic inflammation. In this state, more endogenous ligands are produced, which activate the immune system and impair AT homeostasis [2, 5]. These changes in AT lead to tissue-resident inflammatory/anti-inflammatory immune cell imbalance through an increase in the recruitment of circulating immune cells and a difference in the phenotype of tissue-resident macrophages (inflammatory/antiinflammatory macrophages [M1/M2] polarization) in AT [5-8]. At this state, endogenous ligands (glucose and saturated fatty acid) activate inflammatory pathways like toll-like receptors (TLR) $[9,10]$, considered the key regulators of metabolic disorder progression [1]. As TLR4 becomes activated, signaling transition is conducted by activating the intracellular toll/interleukin (IL)-1 receptor [7] domain, which recruits the important myeloid differentiation factor 88 (MyD88) and TIR domain-containing adaptor-inducing IFN- $\beta$ (TRIF) adaptors. Consequently, these adaptors provoke MyD88- and TRIF-dependent downstream cascades, which in turn activate the nuclear factor (NF)- $\mathrm{kB}$ and interferon regulatory factor 3 (IRF3) inflammatory transcriptional factor. Activation of these signaling pathways is mediated by interaction with the tumor receptor-associated factor 6 (TRAF6) adaptor, which leads to the production of many inflammatory cytokines including IL-6, IL-12, IL-1 $\beta$, interferon $\gamma$ (IFN $\gamma$ ) and tumor necrosis factor $\alpha$ (TNF $\alpha)$. These products are strong inducers of M1 polarization [5] and insulin dysfunction in obese (OB) and IR states [1], intensifying the inflammatory condition $[3,11]$. Although TLR4 signaling is a finely tuned pathway in preventing the enormous immune responses by different negative regulators, like A20, it is assumed that the abnormal activation and/or interaction of these negative regulators could be partly involved in suppression of TLR4 signaling [12-14]. Accordingly, diminishment of AT inflammation and the decrease in M1 polarization due to a reduction of the inflammatory activities of circulating immune cells suggest a treatment strategy at the early stages of inflammation [5, 8].

Entering adulthood in an $\mathrm{OB}$ state increases the mortality risk and, on the contrary, an active lifestyle induces lower rates of TLR4 activation, inflammatory monocytes, and chronic diseases $[4,15,16]$. So, it is believed that regular exercise training could be an effective strategy in inhibiting the progress of inflammation. As a result, exercise therapy has been recommended for the treatment of obesity and the related metabolic disorders as

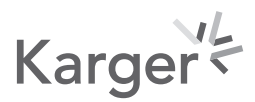


Soltani et al.: Exercise Training, TLR4 Pathway, and Negative Regulators

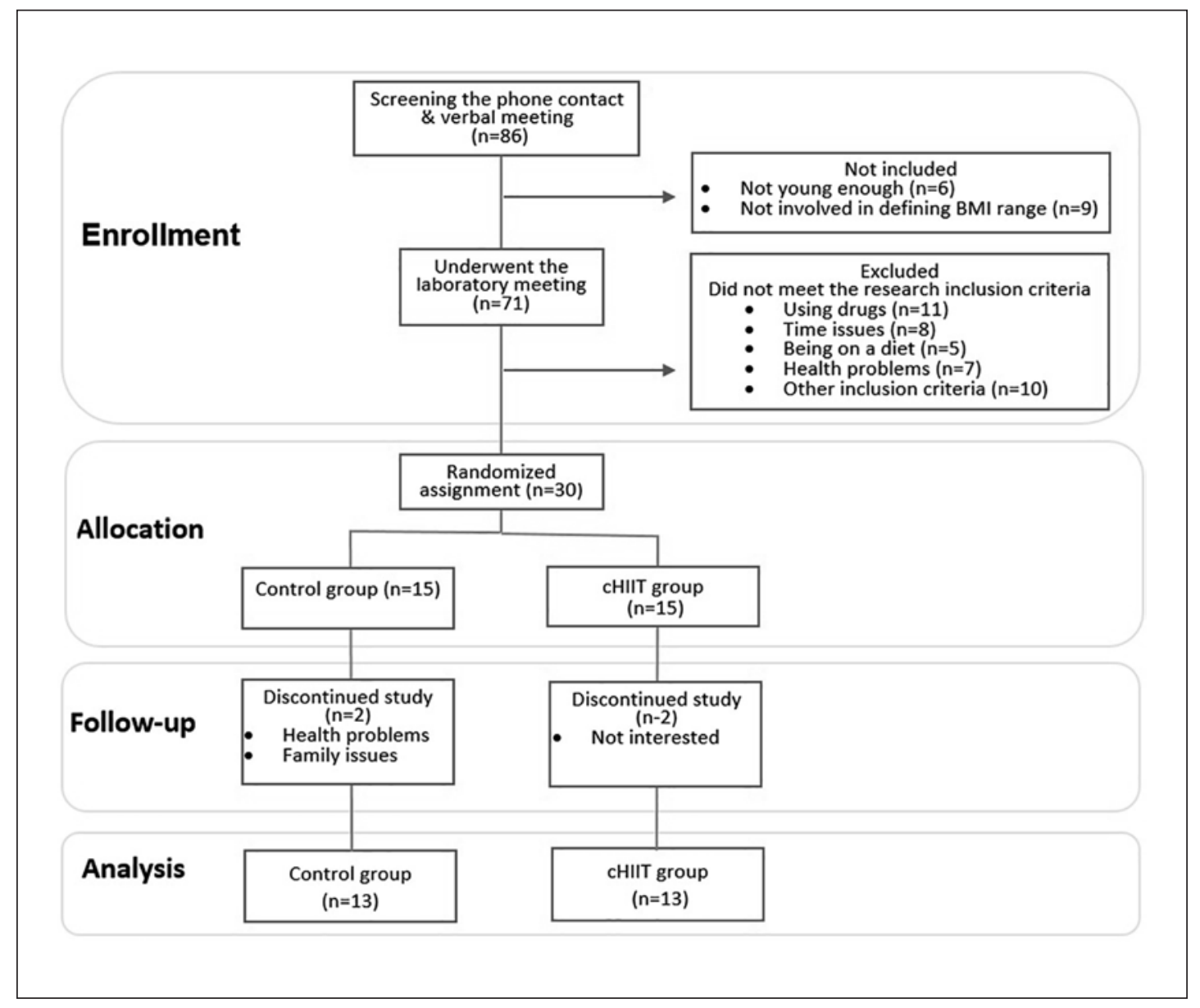

Fig. 1. Flowchart showing the number of subjects at each part of the intervention.

a potent, inexpensive, nonpharmacological approach with minimum side effects $[17,18]$. The immunomodulatory properties of exercise training have indicated a reduction in AT and systemic inflammation [19]. It happens following suppression of macrophage infiltration, an increase in macrophage polarization toward M2 [20,21], and TLR2/4 downregulation in peripheral blood mononuclear cells (PBMCs) [22-25]. However, a lack of time and work-life imbalance constitute the biggest obstacles for participating in exercise training programs [26]. To overcome this phenomenon, new time-efficient exercise protocols like HIIT and all-extremity training have received more attention among researchers. These protocols improve cardiometabolic function through the reduction of TLR2 expression and IR in patients with obesity $[23,27]$. In this context, a few studies which have focused on resistance training (RT) have indicated a low effect on metabolic factors in the youth [28].

In this study, we investigated how a new all-extremity combined HIIT (CHIIT) protocol can change the metainflammatory state. We assessed the TLR4 signaling cascade responses in PBMC of overweight (OW) and $\mathrm{OB}$ females. To the best of our knowledge, there is almost no study relating long-term exercise training to the TLR4 pathway and its adaptors and negative regulators. Knowledge of whether the induced adaptations of CHIIT consisting of both aerobic training (all-extremity cycling) and RT could modify the TLR4 pathway among sedentary young women would be practically beneficial. 


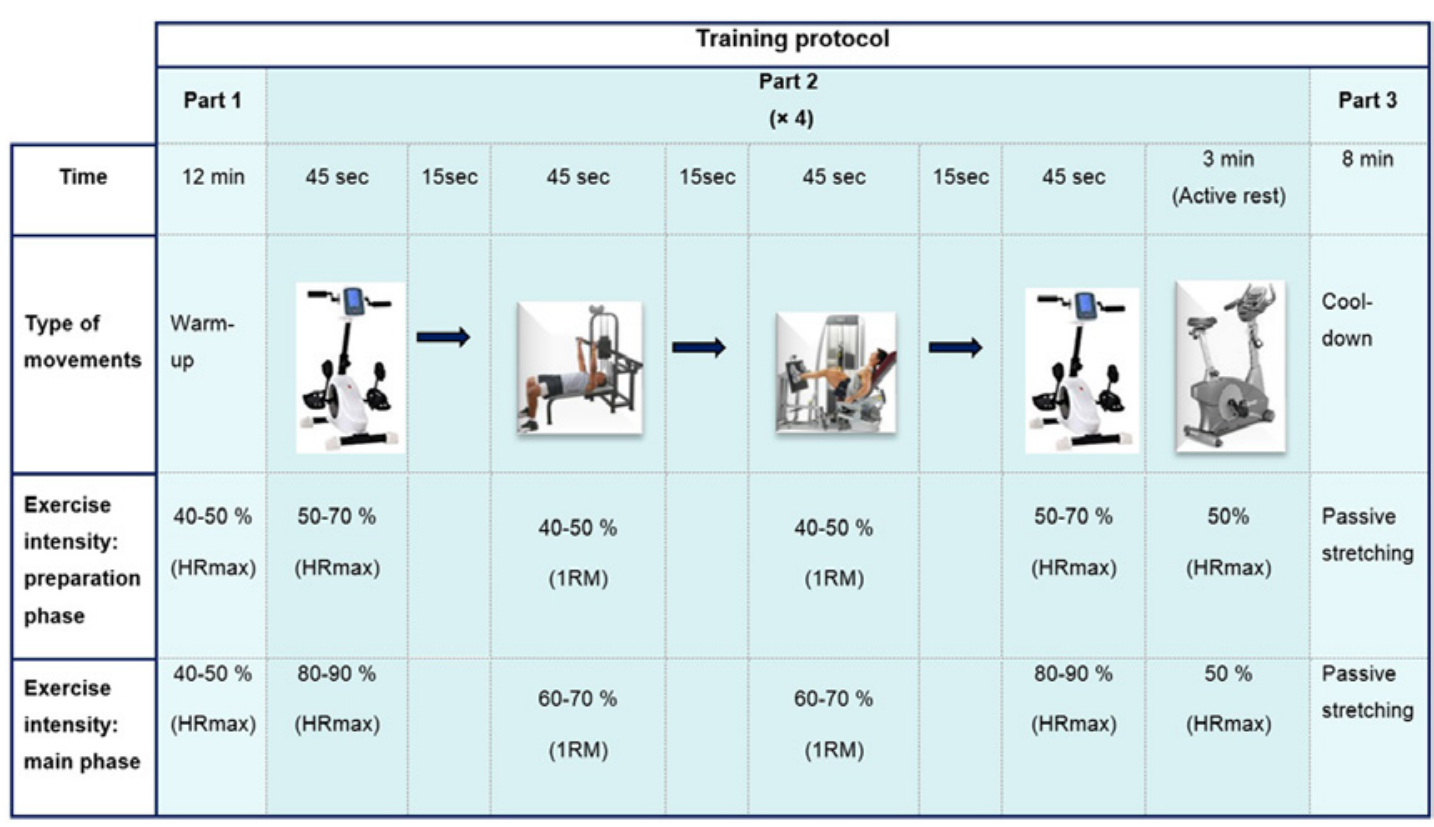

Fig. 2. Schematic figure showing the training protocol, duration, and intensity at each phase and training set.

\section{Methods}

\section{Study Design}

This cross-sectional nonrandomized controlled study design was applied by comparing the control (CG) and exercise groups (EG). The subjects in the EG performed the exercises 4 days a week. All measures were taken first at baseline and then, after 10 weeks (38-40 sessions) of training.

\section{Participants}

All of the participants were recruited via posters posted throughout the University of Isfahan; 86 volunteers were selected for this study (Fig. 1). Seventy-one out of 86 individuals met the inclusion criteria, i.e., healthy young (18-25 years) and OW or OB (BMI = $25-35 \mathrm{~kg} / \mathrm{m}^{2}$ ) patients. As for the exclusion criteria, 41 out of 71 subjects were omitted due to having a nonsedentary lifestyle (participated in any exercise program 6 months before our study), smoking, consuming any medicine, being on a diet, and having cardiovascular diseases (CVD) or kidney, hypertension, IR, and diabetes issues. In the end, a total of 30 eligible women were randomly allocated to the CG $(n=15)$ and the EG $(n=15)$; they were selected from a pool.

\section{Exercise Training Set-Up}

\section{CHIIT Protocol}

All full supervised training sessions (38-40) were performed at the Laboratory of the Sport Science Faculty. The training protocol consisted of a progressive all-extremity CHIT, including 4 sets of 4-min intervals separated by 3 periods of 3-min intervals of active rest (leg cycling) (Figure 2). Each interval involved 4 exercises, i.e., 2 aerobic exercises (arm and leg simultaneous cycling; Monark Ergomedic 839 E and 831 E; Monark, Sweden) and 2 resistance exercises (1 upper body and 1 lower body exercise; Vectra Fitness On-Line 4800; USA) according to a circuit training program where an RT followed all-extremity cycling training. 
The RT part of the protocol, which involved the large muscle groups of the body, consisted of chest presses, lateral pulldowns, leg presses, and leg flexions, where 1 upper and 1 lower body exercise was performed in every interval. The intensity of aerobic training increased gradually according to the age-predicted (220 - age) maximum HR percentage (HRmax) and monitored using a Polar watch (F4 Electro; Oy, Kempele, Finland). The RT load was adjusted according to the one maximum repetition (1RM) percentage. The training intensity and the RT volume increased during the preparation and main phases in a gradual manner. During the preparation phase, in the first 2 weeks, the aerobic training intensity and the RT volume increased from 50 to $70 \%$ HRmax and from 40 to $50 \% 1 \mathrm{RM}$, respectively. The training intensity and volume were reached to $90 \%$ HRmax and 70\% 1RM in the remaining 8 weeks, provided the subjects were able to perform more than 12-15 repetitions of each exercise for 3 consecutive training days, at an increased load of about 5-10\%. Each session began with a warm-up of 10-12 min of cycling, stretching, and kinetic movements and finished with a cooldown consisting of cycling and stretching for $5 \mathrm{~min}$.

Maximal Strength Assessment

The maximal strength of every resistance workout was assessed before the blood testing day in 2 sessions. On the first day, participants were instructed to learn about the proper lifting technique and allowed to practice 2 sets of 8 repetitions. Following the instructions of the 1-6 RM testing procedure, the participants performed the test for all movements on the second day. Finally, strength exercises were evaluated based on the adjusted 1RM\% equation proposed by Nutter [29].

Familiarization with the CHIIT

To become adapted to the laboratory environment and facilities, monitoring the HR, synchronizing the arm and leg cycling, and reaching the target HR were all done during 2 familiarization sessions on 2 nonconsecutive days after the 1RM testing day. Each of the participants performed 2 sets of the protocol, with an increase in the rest time at a 1:1 ratio in the first session, followed by the 2 or 3 intervals of the protocol on the second day.

\section{Measurements}

All of the subjects in both groups were tested twice, i.e., at baseline and in a follow-up run, with the same procedure a day before $1 \mathrm{RM}$ testing and $\sim 48 \mathrm{~h}$ after the last training session to avoid the acute impact of exercise on the test results.

\section{Body Composition and Anthropometric Measures}

Weight and standing height were measured in light clothing without shoes using a Seca 220-gauge stadiometer (Seca, Hamburg, Germany). BMI was calculated as weight (kg) divided by height (m) squared. Waist circumference (WC), hip circumference (HC), and the waist-tohip ratio (WHR) were assessed to indicate the distribution of abdominal fat using an elastic measuring tape. WC and HC were measured between the lowest rib and the iliac crest and in the broadest part of the gluteal muscle, respectively, while participants stood erect [30]. The body fat percent (BF\%) was calculated by measuring the abdominal, triceps, and suprailiac 3 -site subcutaneous skinfold thickness in triplicate using a Lange skinfold caliper (Cambridge Scientific Industries, Cambridge, MD, USA) and the Jackson-Pollock equation for 3-site measurement in women [31].

Cardiorespiratory Fitness

A YMCA submaximal test was run on an electronically braked cycle ergometer (Ergomedic 839 E; Monark, Varberg, Sweden) to determine the subjects' maximal oxygen 
Soltani et al.: Exercise Training, TLR4 Pathway, and Negative Regulators

consumption $\left(\mathrm{VO}_{2 \max }\right)$ [32]. Participants cycled with constant RPM during every stage, while the workload was increased in 3-min intervals. The test was automatically terminated when the HR became constant in 2 consecutive stages at 110-150 bpm. Arterial blood pressure (BP) and resting HR were measured using an automated brachial cuff after $45 \mathrm{~min}$ of seated rest.

Daily Physical Activity and Dietary Monitoring

To ensure that the results of this intervention were not affected by other concurring factors like daily physical activities and dietary changes, the participants were instructed not to change their routine diet or physical activities throughout the study period. Also, to monitor the daily physical activities and dietary energy intake, a pedometer-based step count (AM3; iHealth Labs Inc., USA) and a self-reported 3-day diet recall were applied to record the daily steps and food intake data during the study period on 2 weekdays and one weekend, respectively. These assessments were run at week 1 , the baseline, week 4 or 5 , the middle, and at week 10, the end of the study period. Dietary data were analyzed through NUT 4 software to assess the fixed calorie consumption during the study period.

Blood Sample Preparation

Participants were asked to avoid doing physical activities $48 \mathrm{~h}$ before the blood collection day. After overnight fasting, 12-mL venous blood samples were taken from 7:30 to 9:00 a.m. to prevent circadian variation after a 5-min rest. Leukocytes were isolated from 2-mL heparinized whole blood samples in aliquots using a red blood cell lysis buffer (Biolegend, CA, USA), according to the manufacturer's instructions. The remaining $10 \mathrm{~mL}$ were left to clot to be centrifuged for serum separation. The isolated leukocytes and serum tubes were stored at $-80^{\circ} \mathrm{C}$ until the final analysis.

\section{Gene Expression Assay}

Transcript expression was assessed by quantitative real-time polymerase chain reaction (qPCR) following total RNA extraction from leukocytes using a High Pure RNA Isolation Kit (Roche-life Science, Mannheim, Germany) containing the DNase digestion step to remove the potential genomic DNA residual trace. Following RNA sample quality assessment by spectrophotometry (NanoDrop ${ }^{\mathrm{TM}}$ 2000; Thermo Fisher Scientific, USA), total RNA samples were transcribed in reverse using a BioFact kit (BioFactTM 2X RT Series Pre-Mix; Daejeon, South Korea). The synthesized complementary DNA was used to amplify the gene expression by qPCR (StepOnePlus ${ }^{\mathrm{TM}}$ Real-Time PCR System; Thermo Fisher Scientific, USA). In this step, SYBER Green BioFACT TM 2 X Real-Time PCR Master Mix (Daejeon, South Korea) was consumed. The gene-specific sequences for amplification included:

TLR4 forward: 5'- AAGCCGAAAGGTGATTGTTG -3', reverse: 5'- CTGAGCAGGGTCTTCTCCAC-3'; MyD88 forward: 5' - CTCTCTCCAGGTGCCCATCA -3' ${ }^{\prime}$, reverse: 5' - AGGCGAGTCCAGAACCAAGA -3'; TRIF forward: 5' - GTCCAGGTGTTGGCTCTGTT -3', reverse: 5' - CTGGACGAACACTCCCAGAT - $3^{\prime}$; TRAF6 forward: 5' - CCTTTGGCAAATGTCATCTGTG - ${ }^{\prime}$, reverse: $5^{\prime}$ CTCTGCATCTTTTCATGGCAAC $-3^{\prime} ; N F-\kappa B$ forward: $5^{\prime}$ - GCACGACAACATCTCATTGG $-3^{\prime}$, reverse: $5^{\prime}$ - TCTGCTCCTGCTGCTTTG $-3^{\prime}$; IRF3 forward: $5^{\prime}$ - TCGTGATGGTCAAGGTTGT $-3^{\prime}$, reverse: $5^{\prime}$-TACTGGTCGGAGGTGAGG-3'; $A 20$ forward: 5' - ACAGAAGAGCAACTGAGATC $-3^{\prime}$, reverse: 5'- GTTGGGATGCTGACACTC - $3^{\prime}$; and GAPDH as housekeeping gene forward: $5^{\prime}$ GTGAAGGTCGGAGTCAACGG -3', reverse: 5' - CCTGGAAGATGGTGATGGGAT - 3 '.

All of the experiments were amplified in triplicate, and every run included a nontemplate reaction. The transcript expression values of the target genes were determined in relative units based on the threshold cycle (Ct) of each gene normalized according to GAPDH expression and quantified based on the $2^{-\Delta \Delta \mathrm{Ct}}$ method.

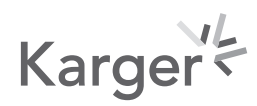


Table 1. Participant characteristics at baseline

\begin{tabular}{lccl}
\hline Variable & CG & EG & $p$ value \\
\hline Anthropometrical variables & & & \\
Age, years & $20.69 \pm 1.54$ & $20.38 \pm 1.5$ & 0.61 \\
Weight, kg & $81.5 \pm 14.69$ & $80.33 \pm 13.25$ & 0.83 \\
& $78(14.75)$ & & \\
BMI & $30.68 \pm 4.24$ & $31.04 \pm 4.16$ & 0.81 \\
& $29.24(5.01)$ & $28.92(4.83)$ & 0.93 \\
$\mathrm{BF}, \%$ & $39.73 \pm 2.13$ & $39.64 \pm 2.81$ & 0.76 \\
WC, cm & $88.88 \pm 10.38$ & $87.73 \pm 8.69$ & \\
Cardiorespiratory fitness & & & 0.77 \\
$\mathrm{RHR}, \mathrm{bpm}$ & $88.84 \pm 11.56$ & $87.53 \pm 11.07$ & 0.02 \\
$\mathrm{SBP}, \mathrm{mm}$ Hg & $112.07 \pm 7.66$ & $105.61 \pm 5.82$ & 0.01 \\
VO $_{\text {2max }}{ }^{\mathrm{a}}, \mathrm{mL} / \mathrm{kg} / \mathrm{min}$ & $26.67 \pm 4.28$ & $30.73 \pm 5.29$ & \\
& & $31.27(6.43)$ & \\
\hline
\end{tabular}

Normally distributed data presented as means \pm SD and nonnormally distributed data are presented as medians (IQR). Differences between the baseline values of 2 groups were analyzed using an independent $t$ test or the Mann-Whitney U test for normally and nonnormally distributed data, respectively. $p$ values were adjusted at $\leq 0.05$. RHR, resting heart rate; SBP, systolic blood pressure; FBS, fasting blood sugar. ${ }^{\text {a Mann- }}$ Whitney U test.

\section{Serum Cytokine Quantification}

The serum cytokine assay was established as a valid approach to assess the final product quantification of the biological pathway. Quantikine ELISA kits were applied to measure the serum levels of TNF $\alpha$ (BioLegend Inc., USA) and IFN $\gamma$ (PeproTech Inc., USA).

\section{Statistical Analysis}

The data distribution was determined by Q-Q plots and the Shapiro-Wilks test; nonnormal data were transformed into a natural logarithm $(\log 10)$. The Levene's test analyzed the homogeneity of variances. Between-group differences at baseline were assessed using an independent $t$ test for normally distributed data or the Mann-Whitney $U$ test for nonnormal distributed data. The dependent variables' responses to the intervention were evaluated by ANCOVA analysis, while the pretesting values were input as the covariate factor. The data were analyzed using a mean differences $t$ test or the Mann-Whitney U test, where the ANCOVA assumptions were violated. The paired sample $t$ test was used to verify within-group changes. The effect size was interpreted via $\Delta \%$ calculation to explore the magnitude of the intervention effect. The Spearman correlation coefficient test was used to assess the correlation of TLR4 and its related adaptors genes with the anthropometrical and cardiorespiratory fitness variables. $p<0.05$ was considered statistically significant. All data are reported means $\pm \mathrm{SD}$ and were analyzed using IBM SPSS Statistics 22 and GraphPad Prism 8.0.1.

\section{Results}

\section{Baseline Characteristics}

The data were analyzed per protocol. Four participants ( 2 from each group) did not complete the training intervention due to family, health, and personal problems, leaving 13 participants per group (Fig. 1). The baseline comparisons of both groups are shown in Table 1. 


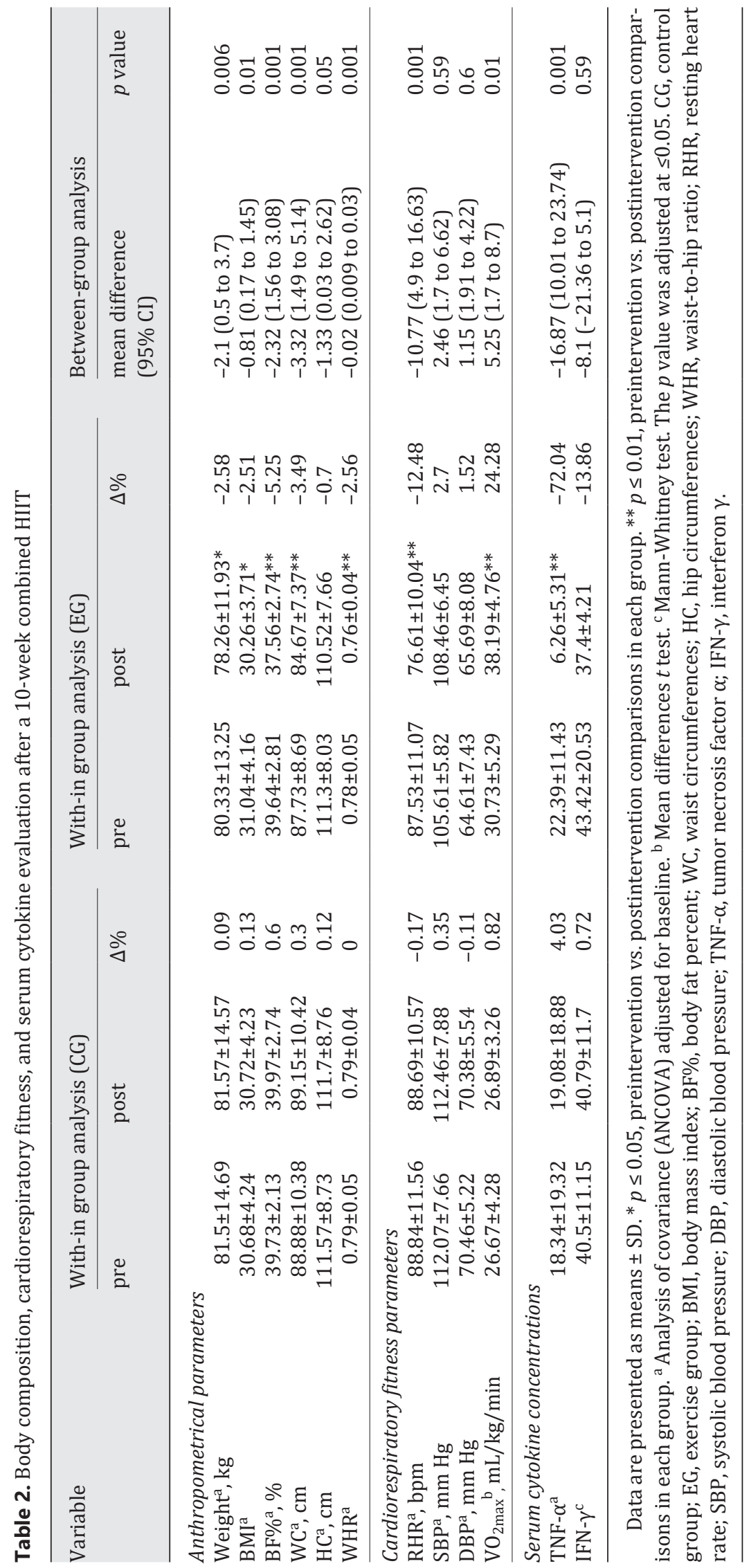


Soltani et al.: Exercise Training, TLR4 Pathway, and Negative Regulators

Table 3. Effect of exercise on the negative regulator, protein adaptors, and transcriptional factors of the TLR4 pathway

\begin{tabular}{|c|c|c|c|c|c|c|c|c|}
\hline \multirow[t]{2}{*}{ Gene } & \multicolumn{2}{|c|}{ With-in group analysis (CG, $\Delta \mathrm{Ct}$ ) } & \multicolumn{2}{|c|}{ With-in group analysis (EG, $\Delta \mathrm{Ct}$ ) } & \multicolumn{4}{|c|}{ Between group analysis $\left(2^{-\Delta \Delta C t}\right)$} \\
\hline & preintervention & postintervention & preintervention & postintervention & CG & $E G$ & $\begin{array}{l}\text { mean difference } \\
(95 \% \mathrm{CI})\end{array}$ & $p$ value \\
\hline TLR4 & $4.21 \pm 2.7$ & $3.22 \pm 2.69 *$ & $2.96 \pm 2.65$ & $4.34 \pm 3.06^{*}$ & $2.25 \pm 1.07$ & $0.84 \pm 1.01$ & $-1.4(-2.24$ to 0.55$)$ & 0.001 \\
\hline MyD88 & $4.55 \pm 4$ & $4.05 \pm 5.06$ & $4.31 \pm 3.19$ & $3.8 \pm 2.34$ & $4.53 \pm 5.15$ & $1.27 \pm 0.88$ & $-3.26(-6.4$ to 0.11$)$ & 0.04 \\
\hline TRIF & $7.01 \pm 3.85$ & $6.38 \pm 3.52^{*}$ & $4.43 \pm 2$ & $3.71 \pm 1.31$ & $1.86 \pm 1.13$ & $3.9 \pm 5.2$ & $2.07(-1.45$ to 5.6$)$ & 0.49 \\
\hline TRAF6 & $5.34 \pm 2.95$ & $4.99 \pm 3.11$ & $5.15 \pm 3.34$ & $4.16 \pm 2.55$ & $2.77 \pm 3.1$ & $2.27 \pm 3.02$ & $-0.49(-3.03$ to 2.05$)$ & 0.69 \\
\hline A20 & $4.97 \pm 5.69$ & $5.51 \pm 5.3$ & $4.94 \pm 5.96$ & $10.4 \pm 5.82^{* *}$ & $0.88 \pm 0.59$ & $0.22 \pm 0.33$ & $-0.66(-1.05$ to -0.27$)$ & 0.002 \\
\hline$N F-\kappa B$ & $6.73 \pm 5.8$ & $6.84 \pm 5.6$ & $3.54 \pm 5.7$ & $7.27 \pm 4.3^{*}$ & $1.61 \pm 2.03$ & $0.23 \pm 0.39$ & $-1.37(-2.67$ to -0.07$)$ & 0.001 \\
\hline IRF3 & $0.28 \pm 6.32$ & $0.33 \pm 5.6$ & $-0.96 \pm 3.61$ & $6.29 \pm 4.58^{* *}$ & $1.22 \pm 0.77$ & $0.25 \pm 0.36$ & $-0.96(-1.46$ to -0.46$)$ & 0.001 \\
\hline
\end{tabular}

Data are presented as means \pm SD. The effect of exercise intervention on gene expression values (EG vs. CG) was analyzed using the formula $2^{-\Delta \Delta C t} * *^{*} \leq 0.05$, preintervention vs. postintervention comparisons in each group. $* * p \leq 0.01$, preintervention vs. postintervention comparisons in each group. CG, control group; EG, exercise group; TLR4, toll-like receptor 4; MyD88, myeloid differentiation factor 88; TRIF, TIR domain-containing adaptor-inducing IFN- $\beta$; TRAF6, tumor receptor-associated factor 6; NF- $\mathrm{B}$, nuclear factor kappa-B; IRF3, interferon regulatory factor 3.

Fig. 3. Three-day recall protocol (a) and daily physical activity (b) data. There were no significant differences in the between- or with-in group analysis in terms of the daily food intake and physical activity in the 2 groups during the study period. 3a

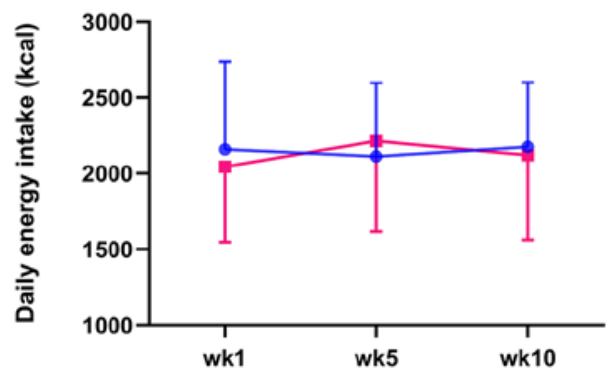

$\rightarrow$ Control

- - Case

$3 b$

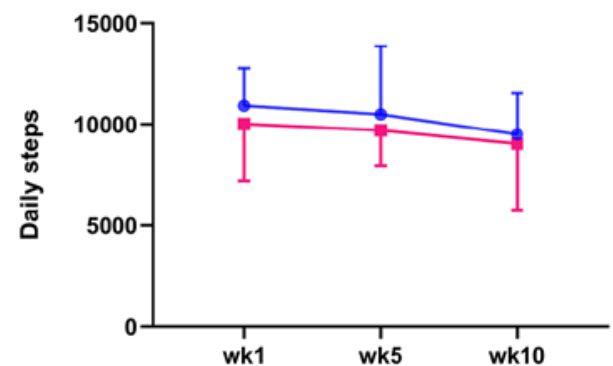

Although there were no statistically significant differences in preintervention anthropometrical variables $(p>0.05)$, the values of systolic blood pressure and $\mathrm{VO} 2_{\max }$ revealed significant differences ( $p=0.02$ and $p=0.01$, respectively). At baseline, the upper and lower levels of mRNA expression of all of the participants were related to TRIF and IRF3, respectively (Table 3).

\section{Daily Energy Intake and Physical Activity}

There was no statistically significant difference $(p>0.05)$ in between- or within-group comparisons in terms of the daily calorie intake (Fig. 3a) and physical activities (Fig. 3b) during the study period. 


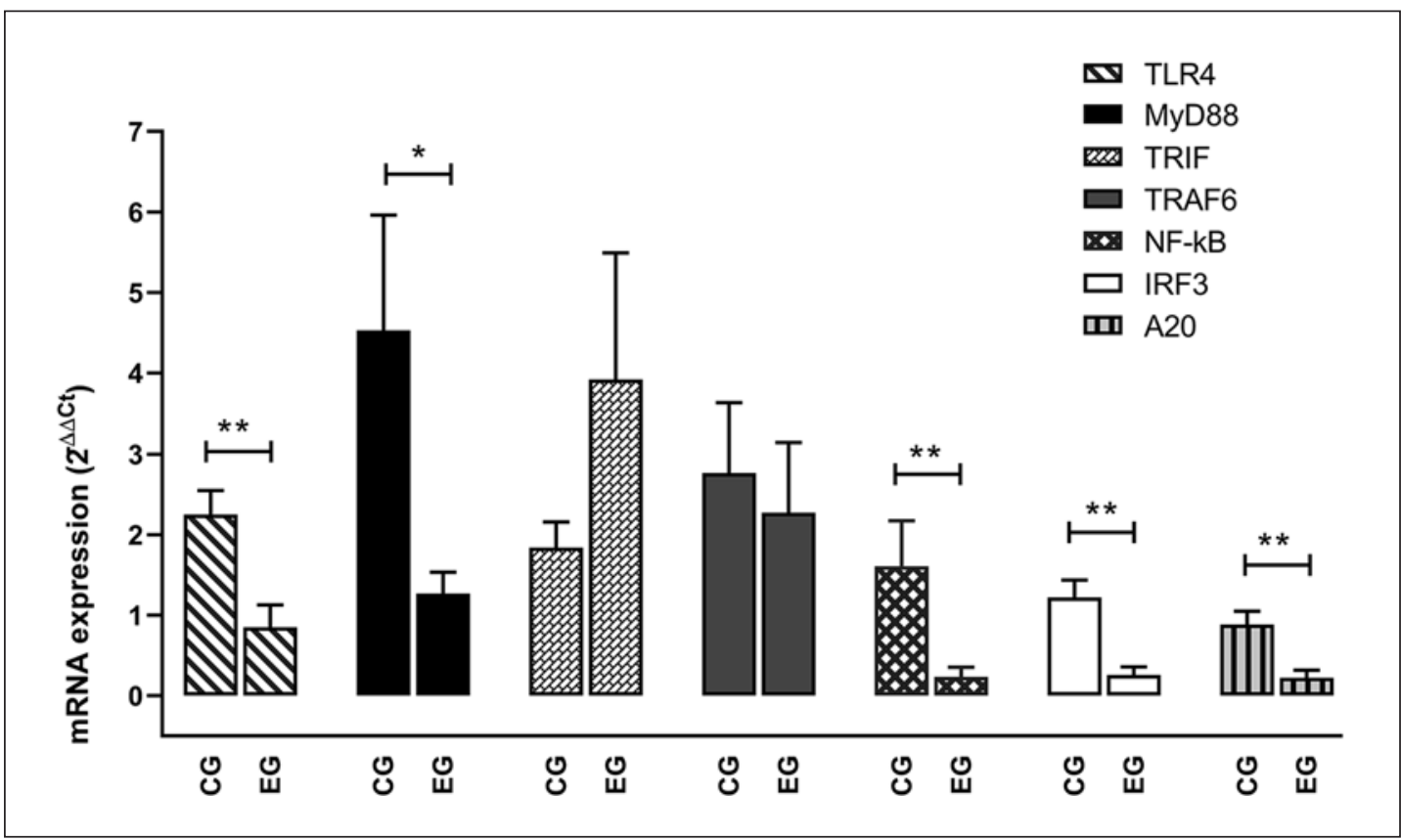

Fig. 4. TLR4 pathway-related adaptors and negative regulator mRNA expression changes in obese subjects following 10-week CHIIT compared to obese CG in PBMC. Values are reported means \pm SEM. ${ }^{*} p<0.05$, $* * p<0.01$ vs. CG.

\section{Body Composition}

Participants in the EG exhibited a statistical significant decrease in weight ( $p=0.006$, $\Delta \%=-2.58)$, BMI $(p=0.01, \Delta \%=-2.51), \mathrm{BF} \%(p=0.001, \Delta \%=-5.25), \mathrm{WC}(p=0.001, \Delta \%=$ $-3.49)$, and WHR ( $p=0.001, \Delta \%=-2.56$ ) either during pre- to posttraining or in comparison to $\mathrm{CG}$, where the $\mathrm{HC}$ changes were not statistically significant compared to the preintervention $(p=0.05)$ and CG ( $p=0.12$; Table 2$)$.

\section{Cardiorespiratory Fitness}

The initial value of $\mathrm{VO}_{2 \max }$ was recorded at preintervention and postintervention; they were $30.73 \pm 5.29$ and $38.19 \pm 4.76 \mathrm{~mL} / \mathrm{kg} / \mathrm{min}$, respectively. Thus, a statistically significant increase of $24.48 \%(p<0.01)$ was found in EG, while no statistically significant improvement was observed in CG. When $\mathrm{VO}_{2 \max }$ was compared in both groups, a significant statistical increase was revealed ( $p=0.01$ ). No statistically significant differences were observed in SPB and DBP between the CG ( $p=0.59$ and $p=0.6$, respectively) and pretesting $(p>0.05)$. Moreover, the resting heart rate improved by $12.48 \%$, which was statistically significant in both within- $(p<0.01)$ and between-group ( $p=0.001)$ comparisons (Table 2$)$.

\section{Serum Cytokines}

$\mathrm{TNF} \alpha$ levels decreased significantly in the EG from preintervention to posttesting $(p<$ $0.01, \Delta \%=-72.04)$ and compared to the CG $(p=0.001)$. Although the IFN $\gamma$ concentration decreased posttesting compared to preintervention $(\Delta \%=-13.86)$, this was not statistically significant $(p>0.05)$ even when compared to CG ( $p=0.59)$ values (Table 2$)$.

\section{TLR4, Adaptors, and Negative Regulator Expression}

According to Figure 4, the CHIIT intervention induced a lower expression of TLR4 in the EG compared to both preintervention $(p<0.05)$ and the CG $(p=0.001)$. Moreover, a signif- 
Soltani et al.: Exercise Training, TLR4 Pathway, and Negative Regulators

Table 4. Correlation coefficients between practical parameters and inflammatory gene expression

\begin{tabular}{|c|c|c|c|c|c|c|c|}
\hline Variable & Weight & BMI & $\mathrm{BF} \%$ & WC & $\mathrm{HC}$ & WHR & $\mathrm{VO}_{2 \text { max }}$ \\
\hline TLR4 & 0.3 & 0.32 & $0.5^{* *}$ & $0.43^{*}$ & 0.23 & $0.45^{* *}$ & -0.28 \\
\hline TRIF & 0.03 & 0.04 & -0.22 & -0.17 & -0.37 & -0.11 & 0.06 \\
\hline MyD88 & -0.08 & -0.09 & 0.27 & 0.12 & -0.03 & 0.14 & -0.13 \\
\hline TRAF6 & -0.19 & -0.23 & -0.03 & -0.13 & -0.17 & -0.03 & 0.14 \\
\hline A20 & 0.24 & 0.25 & $0.62^{* *}$ & 0.33 & 0.22 & $0.42 *$ & -0.13 \\
\hline$N F-\kappa B$ & 0.3 & 0.32 & $0.64^{* *}$ & 0.27 & 0.15 & 0.28 & $-0.45^{*}$ \\
\hline IRF3 & $0.4^{*}$ & $0.39 *$ & $0.61^{* *}$ & $0.56^{* *}$ & 0.41 & $0.55^{* *}$ & -0.11 \\
\hline $\mathrm{TNF} \alpha$ & 0.31 & 0.31 & $0.68^{* *}$ & 0.41 & 0.24 & $0.49 *$ & -0.52 \\
\hline $\mathrm{IFN} \gamma$ & 0.11 & 0.1 & 0.22 & 0.18 & 0.2 & 0.22 & 0.02 \\
\hline
\end{tabular}

${ }^{*} p \leq 0.05$, Spearman correlation test. ${ }^{* *} p \leq 0.01$, Spearman correlation test. TLR4, toll-like receptor 4; MyD88, myeloid differentiation factor 88; TRIF, TIR domain-containing adaptor-inducing IFN- $\beta$; TRAF6, tumor receptor-associated factor 6; NF- $\mathrm{B}$, nuclear factor kappa-B; IRF3, interferon regulatory factor 3; BMI, body mass index; BF\%, body fat percent, WC, waistcircumferences; HC, hip circumferences; WHR, waist-to-hip ratio.

icant statistical decrease was observed at the $M y D 88$ level in the EG compared to the CG ( $p=$ 0.04). However, no statistically significant changes in TRIF or TRAF6 mRNA expression were found in any of the comparisons. NF- $\kappa B$ and IRF3 gene expression significantly decreased after intervention in the EG compared to preintervention ( $p<0.01$ and $p<0.05$, respectively) and the CG $(p=0.001)$. Also, the statistically significant reduction in the $A 20$ mRNA level was found following the intervention in comparison with either the CG $(p<0.01)$ or pretesting $(p=0.002 ;$ Table 3$)$.

\section{Bivariate Comparisons}

According to Table 4, bivariate comparative analyses demonstrated statistically significant positive correlations between TLR4 gene expression changes and BF\% $(r=0.5, p<0.01)$, WC $(r=0.43, p<0.05)$, and WHR $(r=0.45, p<0.01)$. There was a statistical significant positive correlation between IRF3 gene expression changes and weight $(r=0.4, p<0.05)$, BMI $(r=$ $0.39, p<0.05)$, BF\% ( $r=0.61, p<0.01)$, and WHR $(r=0.55, p<0.01)$. Moreover, changes in $N F-\kappa B$ gene expression levels revealed a statistically significant positive correlation with $\mathrm{BF} \%$ $(r=0.64, p<0.01)$ and a negative correlation with $\mathrm{VO}_{2 \max }(r=-0.45, p<0.05)$. A statistically positive association was found between the $A 20$ gene level and BF\% $(r=0.62, p<0.01)$ and WHR $(r=0.42, p<0.05)$. Also, there was a statistically significant positive correlation between the TNF $\alpha$ serum concentration and BF\% $(r=0.68, p<0.01)$ and WHR $(r=0.49, p<0.05)$ values. A statistically significant correlation was found between TRIF, MyD88, TRAF6 genes expression, and all of the variables, as well as the serum concentration of IFN $\gamma$.

\section{Discussion}

To the best of our knowledge, this is the first study to assess the impact of a non-weightbearing combined all-extremity HIIT protocol on the TLR4 pathway (MyD88- and TRIFdependent downstream cascades) and its identical negative regulator. In the present study, we showed that CHIIT downregulated TLR4, MyD88, NF- $\kappa B$, IRF3, and A20 gene expression, while there were no statistically significant differences in TRIF and TRAF6 mRNA expression following 10 weeks of exercise. Serum levels of TNF $\alpha$ and IFN $\gamma$ decreased after the proposed 
Soltani et al.: Exercise Training, TLR4 Pathway, and Negative Regulators

exercise training protocol. Additionally, we found that $\mathrm{VO}_{2 \max }$ was improved, and weight, BMI, BF\%, WC, and WHR were reduced, after CHIIT.

The TLR4 pathway-related adaptors and negative regulator' responses to our CHITT are described in their sequential sense in the following paragraphs.

Animal studies have revealed that high-fat diets increase TLR4 gene levels compared to normal chow [20]. Although the acute protocols have not significantly changed TLR4 mRNA levels, the increase in the duration of training protocols appears to shift these results toward a reduction of TLR4 levels [33]. According to human studies, both acute, though not all, types of exercise (HIIT) [23] and long-term interventions downregulate TLR4 in the obese, healthy, and type 2 diabetes subjects $[24,34,35]$; this corresponds with the findings here. On the contrary, TLR4 levels have remained unchanged or elevated after RT and aerobic protocols $[36,37]$. TLR4 expression decreased in macrophages pretreated with irisin, regarded as a potent anti-inflammatory myokine [38], suggesting the exercise-induced anti-inflammatory myokine as one of the possible mechanisms suppressing TLR4 expression in this study. Accordingly, more experiments are required to resolve these existing contradictions in TLR4 responses to exercise.

As TLR4 recruits both MyD88 and TRIF molecules and consequently activates 2 independent cascades, it is recognized as a prerequisite for obese AT pathology [2,11]. Some RT interventions have reported a reduction in mRNA and protein levels of TRIF [24, 39, 40], while no statistically significant changes were observed in the current study. On the contrary, the MyD88 gene expression level was downregulated in our study, which is supported by previous findings $[24,39,40]$, but not all [36]. It has been reported that the suppression ability of irisin on the TLR4 pathway is associated with inhibition of MyD88 activities [38], suggesting our CHIIT-induced immunomodulatory myokine.

Recruitment of TRAF6 in the activation of 2 TLR4 downstream cascades infers its effects on TLR4 inflammatory power [13]. Consistent with some available studies [39], our protocol did not affect TRAF6 expression, suggesting that this CHIIT might not be strong enough to mediate this adaptor mRNA expression. Upregulation of TRAF6 following exercise was reported by Lira et al. [41] in lipopolysaccharide-treated adipocytes. Accordingly, these conflicts over TRAF6 responses could reveal a regulatory pathway over TRAF6 actions and/ or interactions with other signaling molecules. In this sense, A20 is a negative intracellular regulator of the TLR4 pathway, which induces inhibitory effects on both TRIF- and MyD88dependent cascades by suppressing TRAF6 recruitment [14]. Unlike TRAF6, A20 levels were reduced following our intervention. People with nonalcoholic fatty liver disease and obesity have shown a higher level of A20 expression in the liver in comparison with healthy control individuals $[42,43]$. In contrast to our findings, an acute strenuous exercise intervention has increased the $A 20$ expression levels [44]. To the best of our knowledge, this is the first study to assess A20 responses to a long-term exercise intervention. As adiponectin is one of the proposed proteins inducing beneficial metabolic impacts through A20 [45], it is assumed that some adipo/myokines interfere with A20 expression. More studies are required to explain the exact responses and responsibilities of A20. Therefore, A20 is a possible adaptor in regulating TLR4-induced inflammation in circulating leukocytes during obesity, while its actions and/or interactions in the regulation of TLR4 signaling have remained obscure in response to exercise.

Our intervention reduced the mRNA levels of $N F-\kappa B$ and $I R F 3$, confirming the identical effects of CHIIT on both MyD88- and TRIF-dependent cascades. In the TLR-4 activation process, the expression of different signaling cascade molecules eventually reaches the NF- $\kappa B$ transcription factor which induces production of proinflammatory cytokines such as IL-1 $\beta$, IL-6, and TNF $\alpha$ [3]. So, the reduction of $N F-\kappa B$ and IRF3 gene expression in our study may be due to the decreased TLR-4 expression. Studies have indicated that the gene expression levels

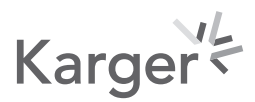


of IRF3, NF- $\kappa B$, and its inhibitors are downregulated in response to both RT and combined interventions $[24,39,40]$, even though continuous aerobic exercise and interval training do not change $N F-\kappa B$ levels [36]. Consistent with our findings the reduction of NF- $\kappa \mathrm{B}$ has been demonstrated in macrophages pretreated with irisin, suggesting a mechanism for exercise in reducing TLR4/NF-kB pathway activity [38]. Moreover, increased secretion of catecholamines, cortisol, and IL- 6 by contracting muscles downregulates the NF- $\kappa$ B pathway [19].

In the present study we found that $\mathrm{TNF} \alpha$ levels were decreased after the proposed exercise training protocol. TNF $\alpha$ has a central role in metabolic dysfunction, and anti-TNF approaches apply as therapy in chronic inflammatory diseases [1]. As we mentioned before, one of the final products of the TLR-4 signaling pathway is TNF $\alpha$, which acts a proinflammatory cytokine. So, it makes sense that by reducing the gene expression of the TLR-4 and then reducing the expression of its downstream molecules in the signaling pathway, such as $I R F 3$ and $N F-\kappa B$, the expression of TNF $\alpha$ as the end product of this pathway will also decrease. A reduction in TNF $\alpha$ serum levels has been shown following long-term protocols [39, 46-48], which is in line with our study, while others have reported increased or unchanged levels [22, $34,49]$. Studies have shown that in the presence of IFN $\gamma$ macrophages shift their phenotype toward M1 and produce proinflammatory cytokines, including IL-12, TNF- $\alpha$, and IL-1 $\beta$ [50, 51]. Also, this cytokine is known as the progenitor of local AT inflammation. Increased IFN $\gamma$ levels during obesity have been associated with the development of M1 polarization and impairment of AT homeostasis during high-fat-diet-induced immunometabolic dysfunction in the early stages [5]. Consistent with previous findings, the IFN $\gamma$ level was decreased following our exercise training protocol $[44,52]$. Although this decrease was not statistically significant, it seems that IFN $\gamma$ reduction effectively decreases the production of TNF- $\alpha$ as an inflammatory cytokine. The other possible mechanism for the decrease in TNF $\alpha$ and IFN $\gamma$ is that contractile muscle-increased IL- 6 develops the production of IL-10. This cytokine as an anti-inflammatory cytokine causes a decrease in TNF $\alpha$ and IFN $\gamma$ levels, suggesting a reduction of M1 polarization in AT, simultaneously [19].

The significant associations between $\mathrm{BF} \%$ and the inflammatory variables, including TNF $\alpha$ serum level and TLR4, NF- $K B$, and IRF3 gene expression, were revealed in this study. Considering that all-cause mortality rates increase by $30 \%$ per $5-\mathrm{kg} / \mathrm{m}^{2}$ increase in BMI [3], the reduction in body composition values in our study revealed the potential benefits of this all-extremity CHIIT for the mortality rate - something that is not supported by an aerobic allextremity protocol [27]. As high-intensity training has been reported to be essential to increase fat oxidation capacity [26], this discrepancy may be due to the differences in the subjects' features and/or the resistance exercises in our protocol.

Regarding an inverse relation between aerobic fitness and inflammation [25], we found a moderate negative correlation between $\mathrm{VO}_{2 \max }$ and $N F-\kappa B$ levels. As the improvement of aerobic fitness per $3.5 \mathrm{~mL} / \mathrm{kg} / \mathrm{min}$ reduces the CVD mortality risk to 19\% [53], the increased level of $\mathrm{VO}_{2 \max }$ in this study (i.e., $24 \%, 5.25 \mathrm{~mL} / \mathrm{kg} / \mathrm{min}$ ) indicates a potential power of our protocol to reduce the CVD mortality risk. These findings are consistent with other studies $[22,47]$. Therefore, prescribing short-duration exercise training can promote health-related physiological aspects and increases the possibility of individuals' participation in exercise training programs.

\section{Study Limitations}

- Because the main focus of this study was on designing a new, potent, time-efficient protocol, the authors did not compare this protocol with traditional exercise training methods.

- Concerning the importance of the same phases of the menstrual cycle for pre- and posttesting results, some participants were in the follicular phase and some were in the luteal 


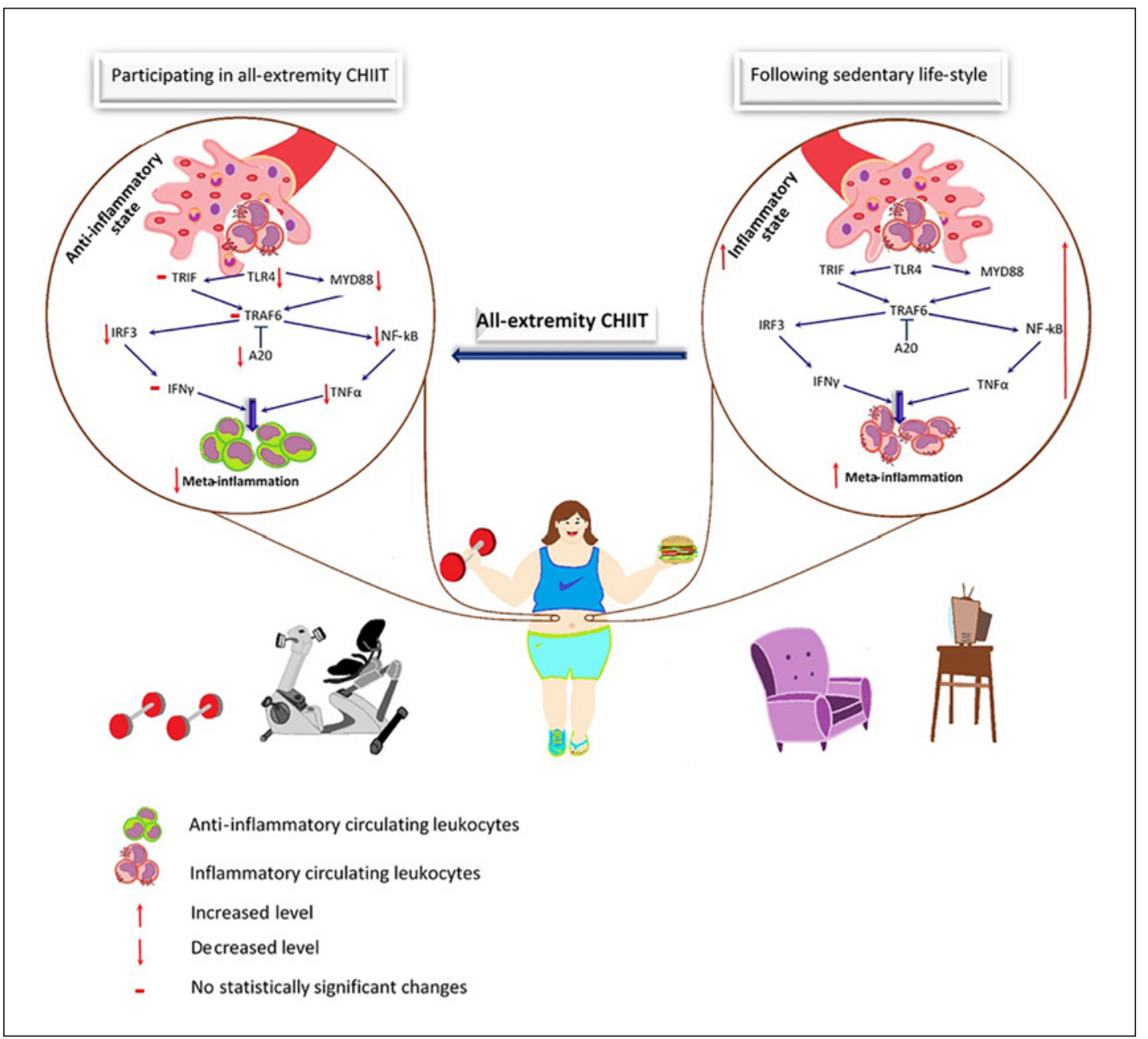

Fig. 5. Changing the circulating leukocyte inflammatory status by exercise training. This proposed all-extremity CHIIT changed the inflammatory state of the circulating leukocytes in the obese individuals by reducing the TLR4 pathway activity. This protocol induced either downregulation of the gene expression of the $T L R 4$ receptor and its downstream adaptors ( $M y D 88$ ), transcriptional factors (IRF3 and $N F-\kappa B$ ), and negative regulator $(A 20)$ or reduction of the final TLR4 product serum levels (TNF $\alpha)$. Overalls, it seems that a 10 -week CHIIT induced an anti-inflammatory state among these young inactive females with obesity.

phase, and the remaining were in other phases. In 10 weeks, these populations confronted abnormalities in their menstrual cycle, preventing control of the same menstrual phase in the testing days.

- Provision of an identical meal for pre- and postintervention blood testing would be more reliable than the subjects' self-reports.

\section{Conclusion}

Overall, our findings point to the immunomodulatory and anti-inflammatory impacts of our all-extremity combined HIIT protocol on metainflammation. These effects were associated with the TLR4 signaling pathway, including downregulation of TLR4, its protein adaptors, transcriptional factors, and negative regulator gene expression or reduction of 
TNF $\alpha$ serum levels. The identical effects of this protocol on both $N F-\kappa B$ and IRF3 were in accordance with A20 downregulation, suggesting that the negative regulators in the TLR4 pathway are the possible mechanisms of exercise for improvement of the inflammatory state in obesity (Fig. 5).

\section{Acknowledgment}

The authors, express their appreciation to Dr. Hossein Khanahmad for his valuable assistance and advice. The support of Mrs. Mohadeseh Toghyani and Mrs. Fahimeh Hosseininasab, from the Immunology Department of the Isfahan University of Medical Science, is appreciated as well.

\section{Statement of Ethics}

This study was approved by the Research Affairs Office, Research Committee of the University of Isfahan, Iran, according to the policy of the Ethics Committee (No. IR.UI. REC.1396.039). All of the subjects underwent a verbal and written check-up by a reliable physician. They were fully informed about the experiment and possible risks before signing a written consent form.

\section{Conflict of Interest Statement}

There are no competing conflicts of interests to declare.

\section{Funding Source}

This study was funded by the University of Isfahan, Isfahan, Iran. Some kits for laboratory experiments were purchased with this financial support.

\section{Author Contributions}

All authors contributed to this study conception. N.S., S.M.M., and N.E. designed this study and performed the statistical analysis. N.S., N.E., and M.K. conducted the laboratory experiments and analyses. N.S. drafted the initial version of this paper, and N.E. edited the final version. All of the authors approved this work.

\section{References}

1 Hotamisligil GS. Inflammation, metaflammation and immunometabolic disorders. Nature. 2017 Feb; 542(7640):177-85.

2 Batatinha HA, Rosa Neto JC, Krüger K. Inflammatory features of obesity and smoke exposure and the immunologic effects of exercise. Exerc Immunol Rev. 2019;25:96-111.

3 Rogero MM, Calder PC. Obesity, Inflammation, Toll-Like Receptor 4 and Fatty Acids. Nutrients. 2018 Mar; 10(4): 432 .

4 Peeters A, Barendregt JJ, Willekens F, Mackenbach JP, Al Mamun A, Bonneux L; NEDCOM, the Netherlands Epidemiology and Demography Compression of Morbidity Research Group. Obesity in adulthood and its consequences for life expectancy: a life-table analysis. Ann Intern Med. 2003 Jan;138(1):24-32. 


\begin{tabular}{l|l}
\hline Obes Facts 2020;13:415-431 \\
\hline DOI: 10.1159/000509132 & $\begin{array}{l}\text { @ 2020 The Author(s). Published by S. Karger AG, Basel } \\
\text { www.karger.com/ofa }\end{array}$ \\
\hline
\end{tabular}

Soltani et al.: Exercise Training, TLR4 Pathway, and Negative Regulators

5 Wensveen FM, Valentić S, Šestan M, Turk Wensveen T, Polić B. The "Big Bang” in obese fat: events initiating obesity-induced adipose tissue inflammation. Eur J Immunol. 2015 Sep;45(9):2446-56.

6 Boutens L, Stienstra R. Adipose tissue macrophages: going off track during obesity. Diabetologia. 2016 May; 59(5):879-94.

7 Pecht T, Gutman-Tirosh A, Bashan N, Rudich A. Peripheral blood leucocyte subclasses as potential biomarkers of adipose tissue inflammation and obesity subphenotypes in humans. Obes Rev. 2014 Apr;15(4):322-37.

8 Yang J, Zhang L, Yu C, Yang XF, Wang H. Monocyte and macrophage differentiation: circulation inflammatory monocyte as biomarker for inflammatory diseases. Biomark Res. 2014 Jan;2(1):1.

9 Dasu MR, Devaraj S, Zhao L, Hwang DH, Jialal I. High glucose induces toll-like receptor expression in human monocytes: mechanism of activation. Diabetes. 2008 Nov;57(11):3090-8.

10 Huang S, Rutkowsky JM, Snodgrass RG, Ono-Moore KD, Schneider DA, Newman JW, et al. Saturated fatty acids activate TLR-mediated proinflammatory signaling pathways. J Lipid Res. 2012 Sep;53(9):2002-13.

11 Akira S, Takeda K. Toll-like receptor signalling. Nat Rev Immunol. 2004 Jul;4(7):499-511.

12 Yang L, Seki E. Toll-like receptors in liver fibrosis: cellular crosstalk and mechanisms. Front Physiol. 2012 May; 3:138.

13 Liew FY, Xu D, Brint EK, O’Neill LA. Negative regulation of toll-like receptor-mediated immune responses. Nat Rev Immunol. 2005 Jun;5(6):446-58.

14 Kondo T, Kawai T, Akira S. Dissecting negative regulation of Toll-like receptor signaling. Trends Immunol. 2012 Sep;33(9):449-58.

15 Walsh NP, Gleeson M, Shephard RJ, Gleeson M, Woods JA, Bishop N, et al. Position statement part one: immune function and exercise. 2011. Available from: https://hdl.handle.net/2134/10584.

16 Booth FW, Roberts CK, Laye MJ. Lack of exercise is a major cause of chronic diseases. Compr Physiol. 2012 Apr; 2(2):1143-211.

17 Giolo De Carvalho F, Sparks LM. Targeting white adipose tissue with exercise or bariatric surgery as therapeutic strategies in obesity. Biology (Basel). 2019 Mar;8(1):16.

18 Pedersen BK, Saltin B. Exercise as medicine - evidence for prescribing exercise as therapy in 26 different chronic diseases. Scand J Med Sci Sports. 2015 Dec;25 Suppl 3:1-72.

19 Gleeson M, Bishop NC, Stensel DJ, Lindley MR, Mastana SS, Nimmo MA. The anti-inflammatory effects of exercise: mechanisms and implications for the prevention and treatment of disease. Nat Rev Immunol. 2011 Aug;11(9):607-15.

20 Kawanishi N, Yano H, Yokogawa Y, Suzuki K. Exercise training inhibits inflammation in adipose tissue via both suppression of macrophage infiltration and acceleration of phenotypic switching from M1 to M2 macrophages in high-fat-diet-induced obese mice. Exerc Immunol Rev. 2010;16:105-18.

21 Oliveira AG, Araujo TG, Carvalho BM, Guadagnini D, Rocha GZ, Bagarolli RA, et al. Acute exercise induces a phenotypic switch in adipose tissue macrophage polarization in diet-induced obese rats. Obesity (Silver Spring). 2013 Dec;21(12):2545-56.

22 Robinson E, Durrer C, Simtchouk S, Jung ME, Bourne JE, Voth E, et al. Short-term high-intensity interval and moderate-intensity continuous training reduce leukocyte TLR4 in inactive adults at elevated risk of type 2 diabetes. J Appl Physiol (1985). 2015 Sep;119(5):508-16.

23 Durrer C, Francois M, Neudorf H, Little JP. Acute high-intensity interval exercise reduces human monocyte Toll-like receptor 2 expression in type 2 diabetes. Am J Physiol Regul Integr Comp Physiol. 2017 Apr; 312(4):R529-38.

24 Rodriguez-Miguelez P, Fernandez-Gonzalo R, Almar M, Mejías Y, Rivas A, de Paz JA, et al. Role of Toll-like receptor 2 and 4 signaling pathways on the inflammatory response to resistance training in elderly subjects. Age (Dordr). 2014;36(6):9734.

25 Lavie CJ, Church TS, Milani RV, Earnest CP. Impact of physical activity, cardiorespiratory fitness, and exercise training on markers of inflammation. J Cardiopulm Rehabil Prev. 2011 May-Jun;31(3):137-45.

26 Gibala MJ, McGee SL. Metabolic adaptations to short-term high-intensity interval training: a little pain for a lot of gain? Exerc Sport Sci Rev. 2008 Apr;36(2):58-63.

27 Hwang CL, Yoo JK, Kim HK, Hwang MH, Handberg EM, Petersen JW, et al. Novel all-extremity high-intensity interval training improves aerobic fitness, cardiac function and insulin resistance in healthy older adults. Exp Gerontol. 2016 Sep;82:112-9.

28 Bea JW, Blew RM, Howe C, Hetherington-Rauth M, Going SB. Resistance Training Effects on Metabolic Function Among Youth: A Systematic Review. Pediatr Exerc Sci. 2017 Aug;29(3):297-315.

29 Nutter J. Weight training adds up. Strategies. 1995;8(7):15-8.

30 Bray GA, Ryan DH. Clinical evaluation of the overweight patient. Endocrine. 2000 0ct;13(2):167-86.

31 YMCA of the USA. YMCA fitness testing and assessment manual. Champaign: Human Kinetics; 2000.

32 Golding LA, Myers CR, Sinning WE. Y's way to physical fitness: the complete guide to fitness testing and instruction. Champaign: Human Kinetics; 1989.

33 Soltani N, Marandi SM, Kazemi M, Esmaeil N. The Exercise Training Modulatory Effects on the Obesity-Induced Immunometabolic Dysfunctions. Diabetes Metab Syndr Obes. 2020 Mar;13:785-810.

34 Slusher AL, Zúñiga TM, Acevedo EO. Maximal Exercise Alters the Inflammatory Phenotype and Response of Mononuclear Cells. Med Sci Sports Exerc. 2018 Apr;50(4):675-83.

35 Liu Y, Liu SX, Cai Y, Xie KL, Zhang WL, Zheng F. Effects of combined aerobic and resistance training on the glycolipid metabolism and inflammation levels in type 2 diabetes mellitus. J Phys Ther Sci. 2015 Jul;27(7):2365-71. 
36 Nickel T, Hanssen H, Emslander I, Drexel V, Hertel G, Schmidt-Trucksäss A et al. Immunomodulatory effects of aerobic training in obesity. Mediators Inflamm. 2011;2011:308965.

37 Markofski MM, Flynn MG, Carrillo AE, Armstrong CL, Campbell WW, Sedlock DA. Resistance exercise traininginduced decrease in circulating inflammatory CD14+CD16+ monocyte percentage without weight loss in older adults. Eur J Appl Physiol. 2014 Aug;114(8):1737-48.

38 Mazur-Bialy AI, Pocheć E, Zarawski M. Anti-Inflammatory Properties of Irisin, Mediator of Physical Activity, Are Connected with TLR4/MyD88 Signaling Pathway Activation. Int J Mol Sci. 2017 Mar; 18(4):701.

39 Fernandez-Gonzalo R, De Paz JA, Rodriguez-Miguelez P, Cuevas MJ, González-Gallego J. TLR4-mediated blunting of inflammatory responses to eccentric exercise in young women. Mediators Inflamm. 2014;2014: 479395.

40 Fernandez-Gonzalo R, De Paz JA, Rodriguez-Miguelez P, Cuevas MJ, González-Gallego J. Effects of eccentric exercise on toll-like receptor 4 signaling pathway in peripheral blood mononuclear cells. J Appl Physiol (1985). 2012 Jun;112(12):2011-8.

41 Lira FS, Rosa JC, Pimentel GD, Seelaender M, Damaso AR, Oyama LM, et al. Both adiponectin and interleukin-10 inhibit LPS-induced activation of the NF-KB pathway in 3T3-L1 adipocytes. Cytokine. 2012 Jan;57(1):98-106.

42 Ai L, Xu Q, Wu C, Wang X, Chen Z, Su D, et al. A20 attenuates FFAs-induced lipid accumulation in nonalcoholic steatohepatitis. Int J Biol Sci. 2015 Nov;11(12):1436-46.

43 Jung UJ, Seo YR, Ryu R, Choi MS. Differences in metabolic biomarkers in the blood and gene expression profiles of peripheral blood mononuclear cells among normal weight, mildly obese and moderately obese subjects. $\mathrm{Br}$ J Nutr. 2016 Sep;116(6):1022-32.

44 Abbasi A, Hauth M, Walter M, Hudemann J, Wank V, Niess AM, et al. Exhaustive exercise modifies different gene expression profiles and pathways in LPS-stimulated and un-stimulated whole blood cultures. Brain Behav Immun. 2014 Jul;39:130-41.

45 Hand LE, Usan P, Cooper GJ, Xu LY, Ammori B, Cunningham PS, et al. Adiponectin induces A20 expression in adipose tissue to confer metabolic benefit. Diabetes. 2015 Jan;64(1):128-36.

46 Lambert CP, Wright NR, Finck BN, Villareal DT. Exercise but not diet-induced weight loss decreases skeletal muscle inflammatory gene expression in frail obese elderly persons. J Appl Physiol (1985). 2008 Aug; 105(2): 473-8.

47 Timmerman KL, Flynn MG, Coen PM, Markofski MM, Pence BD. Exercise training-induced lowering of inflammatory (CD14+CD16+) monocytes: a role in the anti-inflammatory influence of exercise? J Leukoc Biol. 2008 Nov;84(5):1271-8.

48 Phillips MD, Patrizi RM, Cheek DJ, Wooten JS, Barbee JJ, Mitchell JB. Resistance training reduces subclinical inflammation in obese, postmenopausal women. Med Sci Sports Exerc. 2012 Nov;44(11):2099-110.

49 Barry JC, Simtchouk S, Durrer C, Jung ME, Mui AL, Little JP. Short-term exercise training reduces anti-inflammatory action of interleukin-10 in adults with obesity. Cytokine. 2018 Nov;111:460-9.

50 Kang K, Park SH, Chen J, Qiao Y, Giannopoulou E, Berg K et al. Interferon- $\gamma$ represses M2 gene expression in human macrophages by disassembling enhancers bound by the transcription factor MAF. Immunity. 2017 Aug;47(2):235-50.e4.

51 Wang F, Zhang S, Jeon R, Vuckovic I, Jiang X, Lerman A, et al. Interferon gamma induces reversible metabolic reprogramming of M1 macrophages to sustain cell viability and pro-inflammatory activity. EBioMedicine. 2018 Apr;30:303-16.

52 de Souza DC, Matos VA, Dos Santos VO, Medeiros IF, Marinho CS, Nascimento PR, et al. Effects of high-intensity interval and moderate-intensity continuous exercise on inflammatory, leptin, IgA, and lipid peroxidation responses in obese males. Front Physiol. 2018 May; 9:567.

53 Lee DC, Sui X, Artero EG, Lee IM, Church TS, McAuley PA, et al. Long-term effects of changes in cardiorespiratory fitness and body mass index on all-cause and cardiovascular disease mortality in men: the Aerobics Center Longitudinal Study. Circulation. 2011 Dec;124(23):2483-90. 